The authors have reported that $68.3 \%(82 / 120)$ of patients required renal replacement therapy (RRT). RRT would be a confounding factor for comparing the response of terlipressin and noradrenaline due to a decrease in creatinine levels postdialysis. Can the authors clarify regarding the time points used for assessment of response to terlipressin or noradrenaline among those patients who received RRT?

We would also like to highlight an error in figure $3 \mathrm{~A}$. The number mentioned in the third row under the column for day 28 does not match the number shown in the figure (should be 12 instead of 18).

Abhinav Anand, M.D.

Shalimar, M.D., D.M.

Department of Gastroenterology and Human

Nutrition, All India Institute of Medical Sciences, New Delhi, India

\section{REFERENCES}

1) Arora V, Maiwall R, Choudhury A, Jain P, Kumar G, Sarin S, et al. Terlipressin is superior to noradrenaline in the management of acute kidney injury in acute on chronic liver failure. HePatology 2018; doi:10.1002/hep.30208. [Epub ahead of print]

2) Davenport A, Sheikh MF, Lamb E, Agarwal B, Jalan R. Acute kidney injury in acute-on-chronic liver failure: where does hepatorenal syndrome fit? Kidney Int 2017;92:1058-1070.

3) Langenberg C, Bagshaw SM, May CN, Bellomo R. The histopathology of septic acute kidney injury: A systematic review. Crit Care 2008;12:R38.

4) Cecconi M, Evans L, Levy M, Rhodes A. Sepsis and septic shock. Lancet 2018;392:75-87.

(C) 2018 by the American Association for the Study of Liver Diseases.

View this article online at wileyonlinelibrary.com.

DOI 10.1002/hep.30299

Potential conflict of interest: Nothing to report.

\title{
Staging and Restaging for Hepatocellular Carcinoma: Solution of Confusion?
}

\section{TO THE EDITOR:}

We read with interest "Restaging Patients With Hepatocellular Carcinoma Before Additional Treatment Decisions: A Multicenter Cohort Study" by Vitale et al. ${ }^{(1)}$ Although it is an elegant study containing a large cohort of hepatocellular carcinoma (HCC) patients, some concerns should be addressed.

The Italian Liver Cancer (ITA.LI.CA) staging system was proposed by the Italian investigators to predict the outcome of HCC patients. The ITA.LI.CA system uses a scoring method (from 0 to 13) to allocate patients into four prognostic groups. However, in most currently used systems, at least five groups are employed to reflect the highly variable outcome of HCC. A more recent study further indicated that by using clinically available parameters, seven distinct prognostic groups can be identified. ${ }^{(2)}$ Notably, the study by Vitale et al. only compared ITA.LI.CA with the Barcelona Clinic Liver Cancer, the Hong Kong Liver Cancer, and the Cancer of the Liver Italian Program systems. ${ }^{(1)}$ This is apparently not adequate because other important prognostic tools, such as the Japan Integrated System, the Model to Estimate Survival in Ambulatory HCC, and the Model to Estimate Survival in HCC, were not discussed in the study.

Multiple staging systems have been proposed and generally claimed to have better prognostic performance. The lack of consensus may result from the heterogeneous nature of tumor biology and variable treatment strategies at initial staging and restaging. The Child-Turcotte-Pugh (CTP) classification is used in the ITA.LI.CA system. However, a new marker, the albumin-bilirubin (ALBI) grade, as a refinement of the CTP classification, was proposed as a more reliable prognostic marker for HCC. ${ }^{(3)}$ A major disadvantage of CTP classification in $\mathrm{HCC}$ is that many patients who do not have cirrhosis or have only mild cirrhosis are classified as CTP class A. Further discrimination by the ALBI grade showed that it can identify two prognostic groups, implying that not all CTP class A patients are the same. ${ }^{(4)}$ Therefore, it is interesting to know if the ALBI grade, instead of CTP classification, should be used in the ITA.LI.CA system for outcome prediction. In summary, a more comprehensive 
evaluation for the existing staging systems for HCC should be performed to determine which model has the best predictive accuracy. The ALBI grade, as a new player in $\mathrm{HCC}$, may be considered to integrate into the staging system to further enhance the prognostic performance.

Teh-Ia Huo, M.D. ID 1-3
Po-Hong Liu, M.D., M.P.H. ${ }^{2,4}$
Chia-Yang Hsu, M.D., M.P.H. ${ }^{2,5}$
${ }^{1}$ Department of Medicine, Taipei Veterans
General Hospital, Taipei, Taiwan
${ }^{2}$ Faculty of Medicine, National Yang-Ming
University School of Medicine, Taipei, Taiwan
${ }^{3}$ Institute of Pharmacology, National Yang-Ming
University School of Medicine, Taipei, Taiwan
${ }^{4}$ Department of Internal Medicine, University of
Texas Southwestern Medical Center, Dallas, TX
${ }^{5}$ Division of Gastroenterology and
Hepatology, University of Michigan
Ann Arbor, MI

\section{REFERENCES}

1) Vitale A, Farinati F, Noaro G, Burra P, Pawlik TM, Bucci $\mathrm{L}$, et al. Restaging patients with hepatocellular carcinoma before additional treatment decisions: a multicenter cohort study. Hepatology 2018;68:1232-1244.

2) Liu PH, Hsu CY, Hsia CY, Lee YH, Huang YH, Su CW, et al. Proposal and validation of a new model to estimate survival for hepatocellular carcinoma patients. Eur J Cancer 2016;63:25-33.

3) Johnson PJ, Berhane S, Kagebayashi C, Satomura S, Teng M, Reeves HL, et al. Assessment of liver function in patients with hepatocellular carcinoma: a new evidence-based approach-the ALBI grade. J Clin Oncol 2015;33:550-558.

4) Liu PH, Hsu CY, Hsia CY, Lee YH, Chiou YY, Huang YH, et al. ALBI and PALBI grade predict survival for hepatocellular carcinoma across treatment modalities and BCLC stages in the MELD era. J Gastroenterol Hepatol 2017;32:879-886.

Supported by Taipei Veterans General Hospital (VN107-04).

(C) 2018 by the American Association for the Study of Liver Diseases.

View this article online at wileyonlinelibrary.com.

DOI 10.1002/hep.30324

Potential conflict of interest: Nothing to report.

\section{Reply}

We read with great interest the comment letter by Huo et al., because this letter gives us the possibility to better explain some aspects of the ITA.LI.CA prognostic system that were probably unclear in our previous studies. ${ }^{(1,2)}$

First, the ITA.LI.CA system is a score based on 14 prognostic subgroups and not only 4: The lowest score (ITA.LI.CA score $=0$ ) of the model corresponded to the best prognosis, and the highest score (ITA.LI.CA score $=13$ ) was associated with the worst prognosis. In the original paper ${ }^{(2)}$ in which the ITA.LI.CA score was developed and externally validated, and in this paper focused on restaging, ${ }^{(1)}$ we stratified our score in quartiles only to clearly and legibly represent the discrimination and calibration abilities of the ITA.LI.CA system by Kaplan-Meier survival curves.

Second, the ITA.LI.CA score already proved to have a better prognostic performance than JIS and MESIAH scores in two large Italian and Taiwanese cohorts of patients with hepatocellular carcinoma, ${ }^{(2)}$ and in a recent independent Italian validation study. ${ }^{(3)}$ The aim of our recent study ${ }^{(1)}$ was, therefore, not to compare again all the available systems, but only to understand the prognostic behavior of prognostic systems at restaging compared with first prognostic assessment at baseline, and to evaluate how to improve them by adding new prognostic variables available only at restaging (i.e., progressive disease after $1^{\circ}$ therapy, Model for End-Stage Liver Disease at restaging, new therapeutic decision).

It is true, as suggested by Huo et al., that in none of the studies evaluating the ITA.LI.CA score ${ }^{(1-3)}$ has a formal comparison with the Taiwanese MESH score been performed, mainly because of the fact that this score has been proposed simultaneously to the ITA. LI.CA system. The MESH score, however, seems to have some potential limitations in comparison with the ITA.LI.CA score mainly related to a simple dichotomization of some relevant prognostic variables (i.e., tumor burden within vs. beyond Milan criteria, Child score 5 vs. $\geq 6$ ), and to the absence of a strong external validation.

Third, we thank our colleagues for the suggestion to test the ALBI grade within the ITA.LI.CA system. Indeed, in our original paper, ${ }^{(2)}$ we already tested the ALBI grade as a potential variable to include in the ITA.LI.CA score, but its prognostic ability resulted significantly lower than that of the Child Pugh score ${ }^{(2)}$ (Supporting Tables S1 and S2).

\footnotetext{
Alessandro Vitale, M.D., Ph.D. (iD 1

Fabio Farinati, M.D. ${ }^{1}$

Mauro Borzio, M.D. ${ }^{2}$
} 